\title{
Increased serum levels of lipocalin-I and -2 in patients with stable chronic obstructive pulmonary disease
}

This article was published in the following Dove Press journal:

International Journal of COPD

27 May 2014

Number of times this article has been viewed

\author{
Xiao-ru Wangl,* \\ Yong-pu $\mathrm{Li}^{2}, *$ \\ Shui Gao ${ }^{2}$ \\ Wei $\mathrm{Xia}^{2}$ \\ Kun Gao' \\ Qing-hua Kong' \\ Hui Qi' \\ Ling $\mathrm{Wu}^{\prime}$ \\ Jing Zhang ${ }^{3}$ \\ Jie-ming $\mathrm{Qu}^{4}$ \\ Chun-xue $\mathrm{Bai}^{3}$
}

'Department of Pulmonary Medicine, Dahua Hospital, Xuhui District,

Shanghai, ${ }^{2}$ Department of Pulmonary

Medicine, People's Hospital of

Changshou, Chongqing, ${ }^{3}$ Department of Pulmonary Medicine, Zhongshan

Hospital, ${ }^{4}$ Department of Pulmonary Medicine, Huadong Hospital, Fudan

University, Shanghai, People's Republic of China

*These two authors contributed to this paper equally

\begin{abstract}
Despite a number of studies on biomarkers in chronic obstructive pulmonary disease (COPD), only a few disease-related markers have been identified, yet we still have no satisfactory markers specific to innate immune system and neutrophil activation, which is essential in airway inflammation in COPD. Recent biological studies indicated that lipocalins (LCNs) might be involved in airway inflammation and innate immunity; however, results from available studies on the association of LCNs with COPD are not consistent. We carried out a multicenter prospective observational cohort study to investigate the differences in serum levels of LCN1 and LCN2 between subjects with COPD $(n=58)$ and healthy controls $(n=29)$. Several validated inflammatory markers, including $\mathrm{C}$-reactive protein, tumor necrosis factor- $\alpha$, interleukin-6, and interleukin-8, were measured. The correlation of LCN1 and LCN2 with clinical features such as smoking habits, lung function, symptoms, and disease category was also analyzed. When comparing with healthy controls, serum levels of LCN1 $(66.35 \pm 20.26 \mathrm{ng} / \mathrm{mL}$ versus $41.16 \pm 24.19 \mathrm{ng} / \mathrm{mL}, P<0.001)$ and LCN2 $(11.29 \pm 3.92 \mathrm{ng} / \mathrm{mL}$ versus $6.09 \pm 5.13 \mathrm{ng} / \mathrm{mL}$, $P<0.001)$ were both elevated in subjects with COPD after adjusting for age, sex, smoking habits, and inflammatory biomarkers. Smoking history and tobacco exposure, as quantified by pack-year, had no impact on systemic expressions of LCN1 and LCN2 in our study. Blood levels of LCN1 and LCN2, respectively, were negatively correlated to COPD Assessment Test and Modified Medical British Research Council score $(P<0.001)$. Disease category by Global Initiative for Chronic Obstructive Lung Disease grade 1-4 or group A-D was not associated with levels of LCNs. Patient-reported exacerbations and body mass index were also tested, but no relationship with LCNs was found. In summary, serum concentrations of LCN1 and LCN2 were both elevated in patients with COPD, with their levels correlating to COPD Assessment Test and Modified Medical British Research Council score. These findings warrant large-scale and longitudinal studies to validate LCNs as circulating biomarkers for COPD.
\end{abstract}

Keywords: lipocalin-1, lipocalin-2, chronic obstructive pulmonary disease, biomarkers

\section{Introduction}

Chronic obstructive pulmonary disease (COPD) is one of the leading causes of morbidity and mortality both worldwide and in the People's Republic of China, and causes a huge and growing economic and social burden. ${ }^{1-3}$ It is characterized by chronic inflammation and irreversible airflow obstruction, which involves structural changes of the lung. ${ }^{4}$ The heterogeneity of COPD is well acknowledged, but the underlying pathogenesis is still not fully understood. ${ }^{5}$ Circulating biomarkers are easily accessible and would facilitate a deeper understanding of disease mechanism. Blood biomarkers that distinguish subtypes, stratify disease severity and category, predict disease progression, and monitor treatment response would be particularly valuable..$^{6-8}$ 
Despite a number of studies on biomarkers in COPD, only a few disease-related markers have been identified. Inflammatory markers, including C-reactive protein (CRP), fibrinogen, tumor necrosis factor (TNF)- $\alpha$, soluble TNF receptor-1, interleukin-6 (IL-6), and IL-8, are reported to be elevated in COPD and related to lung function and acute exacerbation (AE) ${ }^{9-13}$ Pneumoproteins, including Clara cell secretory protein and surfactant protein $\mathrm{D}$, have recently been identified to be associated with COPD and its clinical phenotypes. ${ }^{14-16}$ These markers, however, are not specific to innate immune system and neutrophil activation, which is essential in airway inflammation in COPD and acute exacerbation of COPD, although IL-6 and IL-8 were acknowledged to be involved in this process. Therefore, it is rational to look into candidate markers from the perspective of innate immune molecules in airways.

Lipocalins (LCNs) are a family of proteins that are involved in the transport of steroids and lipids into cells. Recent studies have suggested that LCNs serve as mediators in airway inflammation. In the LCNs family, LCN1 and LCN2 have been shown to play a role in pulmonary disease and infection. ${ }^{17} \mathrm{LCN} 1$ is expressed in tears, lingual sweat, and lower airway glands, and it can be detected in bronchoalveolar lavage fluid (BALF) and sputum. ${ }^{18,19}$ It is believed that LCN1 is involved in innate immune response against bacterial and fungal infections. It can also inhibit oxidation induced by lipid peroxidation products in vitro. ${ }^{20} \mathrm{LCN} 2$, also named neutrophil gelatinase-associated lipocalin, is constitutively expressed in neutrophilic granules and thus could serve as a marker for neutrophil activity. ${ }^{21}$ It is currently widely used to detect and monitor acute renal failure, indicating a potential value in its application in COPD. ${ }^{22}$ In addition, LCN2 might serve as a mediator in the inflammation network. It can be unregulated in airway structural and inflammatory cells by stimuli like lipopolysaccharide, TNF- $\alpha$, IL-1, and IL-17. ${ }^{17}$ An increased mortality due to Escherichia coli infection in LCN2 knockout mice suggested an antimicrobial function by sequestrating iron. ${ }^{23}$ Most excitingly, Nicholas et al ${ }^{18}$ discovered a reduced sputum level of LCN1, and Eagan et $\mathrm{al}^{24}$ reported an increased plasma concentration of LCN2 in COPD patients recently. Taking these facts together, LCNs might have a potential role as a more specific indicator of innate immunity and neutrophil inflammation. However, the relationship of LCN1 and LCN2 with COPD and disease presentation and severity is still to be validated.

To address this issue, we undertook a multicenter prospective observational cohort study to investigate the differences in systemic levels of LCN1 and LCN2 between subjects with COPD and healthy controls. The association of LCN1 and LCN2 with clinical features such as tobacco exposure, lung function, symptoms, disease category, and inflammatory markers was also analyzed.

\section{Methods \\ Ethics statement}

This study was approved by the Ethics Committee of Zhongshan Hospital of Fudan University, Shanghai, People's Republic of China, and has been registered in the Chinese Clinical Trials Registry (ChiCTR-OCC-11001621). Written informed consent was obtained from all participants.

\section{Definitions}

The diagnosis of COPD was according to the Global initiative for chronic Obstructive Lung Disease (GOLD) definition. Briefly, COPD was considered to be present if postbronchodilator forced expiratory volume in 1 second $\left(\mathrm{FEV}_{1}\right)$ /forced vital capacity (FVC) ratio was less than $0.70 .{ }^{1}$ The grading of COPD was based on $\mathrm{FEV}_{1} / \%$ pred. ${ }^{1} \mathrm{COPD}$ severity was also evaluated according to an $\mathrm{A}, \mathrm{B}, \mathrm{C}$, or D classification proposed by GOLD. ${ }^{1}$

Nonsmokers were defined as never smokers or exsmokers with less than 5 pack-years of tobacco exposure and having quit for at least 10 years; otherwise, the subjects were classified as smokers.

\section{Study population and subject enrollment}

Participants in this study were recruited from Zhongshan Hospital of Fudan University (a comprehensive teaching hospital) and Dahua Hospital (a district hospital) in Shanghai, and Changshou People's Hospital (a district hospital) in Chongqing, People's Republic of China, since December 2011. The last subject was enrolled in October 2012 when the estimated sample size was achieved. Healthy subjects without COPD were recruited as controls.

Participants who had a primary diagnosis of congestive heart failure, bronchiectasis, bronchial asthma, active pulmonary tuberculosis, obstructive bronchiolitis, pneumosilicosis, interstitial lung disease, pleural effusion, diffuse panbronchiolitis, or a history of pneumonectomy were excluded from the study. Patients who had airflow limitation due to abnormalities in large airways were also excluded. Those who had a previous exacerbation of COPD within 4 weeks of admission were excluded.

Subjects with COPD were predominantly recruited from respiratory outpatient clinics and discharged patients from a respiratory ward. Adults aged 45 years and over with a 
history of spirometry-defined physician-diagnosed stable COPD were screened for further enrollment.

\section{Study procedure and data collection}

For all participants, clinical data, including age, sex, weight, height, smoking status (never smoker, ever smoker, or current smoker), quantity of tobacco smoking exposure (pack-years), history of respiratory diseases, respiratory symptoms, number of exacerbations in the previous year, comorbid diseases, and family history, were recorded into a case report form. Spirometry was performed according to international guidelines using a calibrated Jaeger ${ }^{\circledR}$ MasterScreen ${ }^{\mathrm{TM}}$ PFT spirometer (Jaeger, North Rhine-Westphalia, Germany) before and after inhalation of $400 \mathrm{mcg}$ of salbutamol via spacer. Serum was collected at the time of the study visit for measurement of CRP and biomarkers. All the blood samples were collected in the early morning after fasting for at least 8 hours and before taking medications. Subjects with COPD were also asked to complete a COPD Assessment Test (CAT) and a modified Medical British Research Council (mMRC) dyspnea questionnaire.

\section{Quantification of biomarkers of interest in serum samples}

Commercial enzyme-linked immunosorbent assay kits from USCN Life Science Inc. (Wuhan, People's Republic of China) were used for measuring IL-6, IL-8, TNF- $\alpha$, LCN1, and LCN2. The level of protein of interest in serum was determined according to the manufacturer's instructions. Specifically for LCN1 and LCN2, the sensitivity is $25 \mathrm{pg} / \mathrm{mL}$ and the detection range is $78-5,000 \mathrm{pg} / \mathrm{mL}$. The samples were diluted to fit into the linear detection range as needed. The percentage of coefficient of variance of LCN1 and LCN2 was $4.6 \%$ and $5.7 \%$, respectively.

\section{Statistical analysis}

Statistical analyses were performed using SPSS version 19.0 (IBM Corporation, Armonk, NY, USA). Group-wise comparisons using all subjects were performed, and correlation analyses were carried out in COPD patients only. The D'Agostino-Pearson omnibus normality test was performed on continuous data. All continuous data were expressed and graphed as mean \pm standard deviation. For data normally distributed, unpaired $t$-tests or one-way analysis of variance followed by Bonferroni post-test was performed to determine whether the differences between the groups were statistically significant. Otherwise, comparisons were made using the Mann-Whitney test or Kruskal-Wallis analysis followed by Dunn's multiple comparison test. Correlation analyses were carried out using Pearson or Spearman methods, depending on the normality of the data distribution. For categorical variables, a chi-squared test and Spearman rank correlation analysis were used. Multiple logistic regressions were used for multivariate analysis. Differences were considered significant at the level of $P<0.05$.

\section{Results}

\section{Basic characteristics}

In this study, 58 patients with COPD and 29 healthy controls were recruited and tested. The demographic data, smoking habits, grade stratified by postbronchodilator $\mathrm{FEV}_{1}$, and disease category in the context of GOLD A-D are described in Tables 1 and 2. Subjects with COPD smoked more heavily than controls. Although the percentage of males was higher in the COPD group, the difference was not statistically significant. There was no statistically significant difference in serum creatinine levels or glomerular filtration rate between the two groups.

\section{Inflammatory biomarkers}

As shown in Table 3, serum levels of CRP, IL-6, IL-8, and TNF- $\alpha$ were all significantly elevated in patients with COPD compared with in subjects without COPD.

\section{Lipocalins}

As compared with non-COPD controls, serum LCN1 was elevated in subjects with COPD $(66.35 \pm 20.26 \mathrm{ng} / \mathrm{mL}$ versus [vs] $41.16 \pm 24.19 \mathrm{ng} / \mathrm{mL}, P<0.001)$, and levels of LCN2 were also higher $(11.29 \pm 3.92 \mathrm{ng} / \mathrm{mL}$ vs $6.09 \pm 5.13 \mathrm{ng} / \mathrm{mL}$, $P<0.001$ ) (Figure $1 \mathrm{~A}$ and C). LCN1 and LCN2 were significantly higher in the COPD group than in both nonsmoker controls and smoker controls $(P<0.001)$. In multiple logistic regression models, the differences in both LCN1 and LCN2 between the two groups remained significant after adjusting

Table I Basic characteristics of the study population (data expressed as mean \pm standard deviation)

\begin{tabular}{llll}
\hline & Controls & COPD & P-value \\
\hline $\mathrm{n}$ & 29 & 58 & \\
Male/female & $18 / 11$ & $45 / 13$ & 0.131 \\
Age, years & $74.5 \pm 7.9$ & $73.1 \pm 13.0$ & 0.568 \\
Smoker/nonsmoker & $12 / 17$ & $40 / 18$ & 0.016 \\
Pack-year & $10.8 \pm 15.8$ & $31.0 \pm 32.8$ & 0.005 \\
FEV $_{1}$, postbonchodilator & $1.627 \pm 0.615$ & $0.944 \pm 0.34 \mid$ & $<0.001$ \\
FEV $_{1} \%$ pred, postbronchodilator & $71.5 \pm 5.0$ & $48.3 \pm 19.5$ & $<0.001$ \\
FEV $_{1} /$ FVC\% & $80.1 \pm 9.3$ & $53.9 \pm 10.4$ & $<0.001$ \\
\hline
\end{tabular}

Abbreviations: COPD, chronic obstructive pulmonary disease; $\mathrm{FEV}_{1}$, forced expiratory volume in I second; FVC, forced vital capacity; \%pred, percentage predicted. 
Table 2 Severity distribution of subjects with COPD

\begin{tabular}{lll}
$\begin{array}{l}\text { Grade as classified } \\
\text { by lung function, } \\
\text { n (\%) }\end{array}$ & $\begin{array}{l}\text { Group as classified comprehensively } \\
\text { by lung function, symtoms, and acute } \\
\text { exacerbation frequencies, } \mathbf{n}(\%)^{\mathbf{a}}\end{array}$ \\
\hline Grade I & $5(8.6)$ & Group A I $(1.7)$ \\
Grade 2 & $22(37.9)$ & Group B $3(5.2)$ \\
Grade 3 & $23(39.7)$ & Group C $\quad 10(17.2)$ \\
Grade 4 & $8(13.8)$ & Group D $42(7.2)$
\end{tabular}

Note: a Group A is typically GOLD grade I or 2 and/or $\leq$ I exacerbations per year and $\mathrm{mMRC}$ score $<2$ or CAT score $<10$; group $B$ is GOLD grade I or 2 and/or $\leq 1$ exacerbations per year and $\mathrm{mMRC}$ score $\geq 2$ or CAT score $\geq 10$; group $C$ is GOLD grade 3 or 4 and/or $\geq 2$ exacerbations per year and $m M R C$ score $<2$ or CAT score $<10$; group $D$ is GOLD grade 3 or 4 and/or $\geq 2$ exacerbations per year and $m M R C$ score $\geq 2$ or CAT score $\geq 10$.

Abbreviations: CAT, COPD Assessment Test; COPD, chronic obstructive pulmonary disease; GOLD, Global initiative for chronic Obstructive Lung Disease; mMRC, modified Medical British Research Council dyspnea questionnaire.

for age, sex, smoking habit, smoking quantity (pack-year), and inflammatory biomarkers, including CRP, IL-6, IL-8, and TNF- $\alpha$.

In our cohort, smoking history and exposure, as quantified by pack-year, seemed to have no impact on systemic expressions of LCN1 and LCN2. In subgroup analysis of both controls and patients with COPD, no differences in either LCN1 or LCN2 were found between smokers and nonsmokers (data not shown). No relationship between pack-year and levels of LCN1 and LCN2 was observed in COPD subjects (Table 4).

In patients with COPD, bivariate associations between specific disease characteristics and serum levels of LCN1 and LCN2 were investigated. Results are shown in Table 4 and Figure 2. Serum levels of LCNs were negatively correlated to CAT and mMRC score. After adjustment for age, sex, smoking habit, and inflammatory marker, CAT and mMRC scores were still significantly associated with levels of LCN1 and LCN2, respectively. Levels of LCNs in patients of grade 2 and 3 seemed to be lower than in patients of grade 1, but the differences were not statistically significant (Figure 1B and D). Neither $\mathrm{FEV}_{1} / \%$ pred nor $\mathrm{FEV}_{1} / \mathrm{FVC}_{0}$ was related to levels of LCNs. For COPD patients, disease category by GOLD grade $1-4$ or group A-D was not associated with level

Table 3 Comparisons of inflammatory biomarkers between COPD and controls (data expressed as mean \pm standard deviation)

\begin{tabular}{lccc}
\hline & Controls & COPD & P-value \\
\hline CRP $(\mathrm{mg} / \mathrm{L})$ & $3.86 \pm 3.75$ & $7.50 \pm 5.46$ & $0.02 \mathrm{I}$ \\
$\mathrm{IL}-6(\mathrm{pg} / \mathrm{mL})$ & $23.4 \mathrm{I} \pm 12.86$ & $39.2 \mathrm{I} \pm 27.34$ & 0.036 \\
$\mathrm{IL}-8(\mathrm{pg} / \mathrm{mL})$ & $21.70 \pm 18.6 \mathrm{I}$ & $47.12 \pm 30.00$ & $0.00 \mathrm{I}$ \\
$\mathrm{TNF}-\alpha(\mathrm{pg} / \mathrm{mL})$ & $73.46 \pm 50.44$ & $\mathrm{I} 12.7 \pm 80.20$ & $0.07 \mathrm{I}$ \\
\hline
\end{tabular}

Abbreviations: COPD, chronic obstructive pulmonary disease; CRP, C-reactive protein; IL, interleukin; TNF- $\alpha$, tumor necrosis factor- $\alpha$. of LCNs. Patient-reported AEs, body mass index, and blood concentration of TNF- $\alpha$ were also tested, but no relationship with LCNs was found.

\section{Discussion}

Our study confirms that circulating levels of LCN1 and LCN2 were both increased in patients with COPD. It is the first study testing serum LCN1 and LCN2 simultaneously in a Chinese population. As compared with the available data in a similar topic, ${ }^{18,24,25}$ our study involved all of the disease categories as defined by GOLD. In addition to lung function, disease severity was also evaluated using GOLD grouping criteria updated in 2011. ${ }^{1}$ In this context, symptoms were quantified, and this study provided the first piece of information on the negative correlation of serum LCNs with CAT and mMRC score. Participants were recruited prospectively and no data were missing. Considering the role of LCNs in immunity and inflammation, systemic LCN1 and LCN2 were tested along with several validated inflammatory markers to exclude potential confounding factors.

In our study, serum LCN1 was elevated in COPD patients as expected. Our finding was in line with several previous human studies on LCN1. LCN1 was found to be significantly upregulated in saliva, nasal lavage fluid, and BALF in heavy smokers compared with in nonsmokers. ${ }^{19,26}$ It was also increased in bronchial secretions of cystic fibrosis patients compared with healthy subjects. ${ }^{27}$ On the other hand, the only available study on COPD reported a reduction of LCN1 in induced sputum in patients when comparing with healthy smokers, with its levels correlating to $\mathrm{FEV}_{1} / \mathrm{FVC} \%{ }^{18}$ The authors suggested that a decrease in local LCN1 might impair epithelial immune defense and increase risk for infections. Simultaneous measurements of LCN1 in blood and sputum could help to elucidate the controversy.

In previous studies, LCN2 has been found to be elevated in BALF, ${ }^{28}$ induced sputum, ${ }^{29}$ and blood, ${ }^{24,25}$ and this is consistent with our results. Research by Eagen et al. ${ }^{24}$ revealed that LCN2 levels were increased in patients who reported frequent AEs requiring oral steroids, while decreased in those with GOLD grade 3-4 compared with stage 2 and those using inhaled steroids. ${ }^{24} \mathrm{We}$ also note a decrease in the levels of LCN2 in GOLD stage 2-3 patients, but the differences did not reach statistical significance. LCN2 might have multifaceted functions of inflammation enhancement and infection protection, thus explaining the inverted decrease in an advanced stage of disease. In addition, airway bacterial colonization or exacerbation induced by infection might have a mutual impact on LCN expressions. A longitudinal 
A

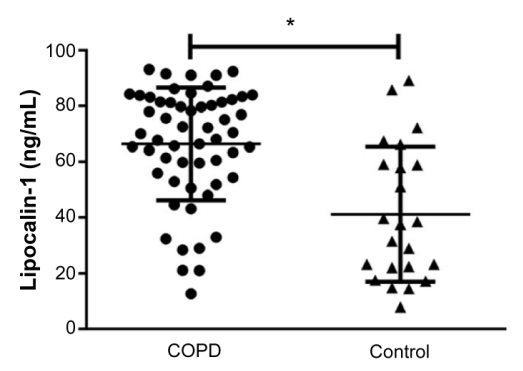

C

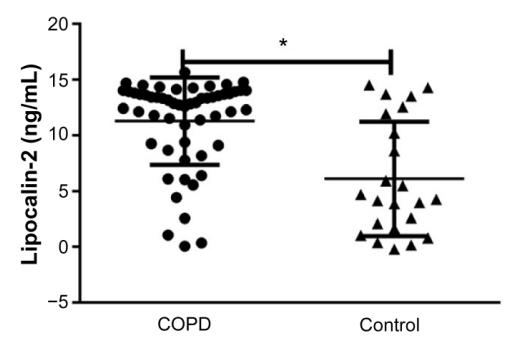

B

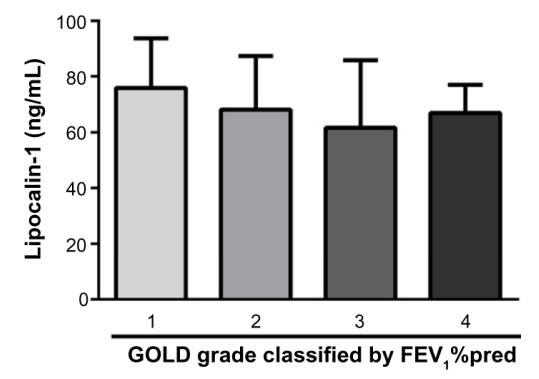

D

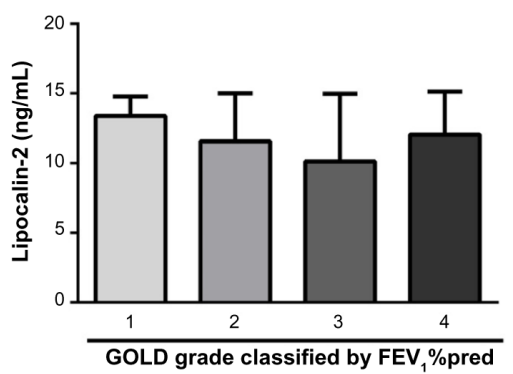

Figure I Comparisons of lipocalins between COPD and control. (A) Serum levels of lipocalin-I. (B) Serum levels of lipocalin-I in patients with GOLD grade I-4 COPD. (C) Serum levels of lipocalin-2. (D) Serum levels of lipocalin-2 in COPD patients with different disease grade. Data are presented as mean \pm standard deviation.

Note: $* P<0.001$.

Abbreviations: COPD, chronic obstructive pulmonary disease; FEV , forced expiratory volume in I second; GOLD, Global initiative for chronic Obstructive Lung Disease; \%pred, percentage predicted.

follow-up of local and systemic levels of LCN2 would clarify the dynamic expression of LCN2 in disease progression and make it a candidate biomarker for disease monitoring. At the same time, LCN2 has been reported to be involved in the pathogenesis of allergic airway inflammation and could be

Table 4 Correlation analysis between serum levels of lipocalins and disease features in patients with COPD

\begin{tabular}{|c|c|c|c|c|}
\hline & \multicolumn{2}{|c|}{$\begin{array}{l}\text { Lipocalin- I } \\
\text { (ng/mL) }\end{array}$} & \multicolumn{2}{|c|}{$\begin{array}{l}\text { Lipocalin-2 } \\
\text { (ng/mL) }\end{array}$} \\
\hline & $r$ & $P$-value & $r$ & $P$-value \\
\hline Smoking (pack-year) & 0.146 & 0.284 & 0.016 & 0.906 \\
\hline FEV \%pred & 0.048 & 0.724 & 0.183 & 0.178 \\
\hline GOLD grade I-4 & -0.185 & 0.173 & -0.153 & $0.26 I$ \\
\hline $\mathrm{FEV}_{1} / \mathrm{FVC} \%$ & -0.005 & 0.972 & -0.057 & 0.679 \\
\hline CAT score & -0.578 & $<0.001$ & -0.534 & $<0.00$ I \\
\hline mMRC score & -0.272 & $<0.001$ & -0.301 & $<0.001$ \\
\hline GOLD group $A-D$ & -0.081 & 0.555 & -0.191 & 0.158 \\
\hline $\begin{array}{l}\text { Number of AEs } \\
\text { in the previous year }\end{array}$ & 0.081 & 0.553 & 0.126 & 0.355 \\
\hline TNF- $\alpha(p g / m L)$ & 0.024 & 0.865 & 0.113 & 0.418 \\
\hline BMI & -0.029 & 0.833 & 0.152 & 0.264 \\
\hline
\end{tabular}

Abbreviations: AEs, acute exacerbations; BMI, body mass index; CAT, COPD Assessment Test; COPD, chronic obstructive pulmonary disease; FEV , forced expiratory volume in I second; FVC, forced vital capacity; GOLD, Global initiative for chronic Obstructive Lung Disease; mMRC, modified Medical British Research Council dyspnea questionnaire; \%pred, precentage predicted; TNF- $\alpha$, tumor necrosis factor- $\alpha$. increased in patients with asthma and airway hypersensitivity, which could be overlapped in patients with COPD. ${ }^{17}$

One of the most striking findings of our study was the relationship of LCNs to symptomatic scores. The only study looking into this issue is by Eagan et al..$^{24}$ The authors reported that respiratory symptoms, which were defined as chronic cough, phlegm, or dyspnea, were not significantly associated with plasma levels of LCN2. Instead of traditional evaluation methods, we adopted CAT and mMRC dyspnea score, with convenience and validity confirmed in Chinese patients, to quantify symptoms, and showed that more symptomatic patients had lower serum levels of LCNs. Eagan et $\mathrm{a}^{24}$ reported that frequent exacerbations, another key component of COPD subtype, were related to higher levels of LCN2. Combined with our data, it indicates that LCNs could be potential indicators for defining subtypes. Further research on biological functions of LCNs is warranted to connect these changes to underlying mechanisms, and studies should be performed to include other parameters of disease features.

This study has limitations. First, it is a study with moderate size, and sample sizes across disease categories were unbalanced, which affected the power to detect associations with modest effect. Furthermore, this study was performed as a 
A

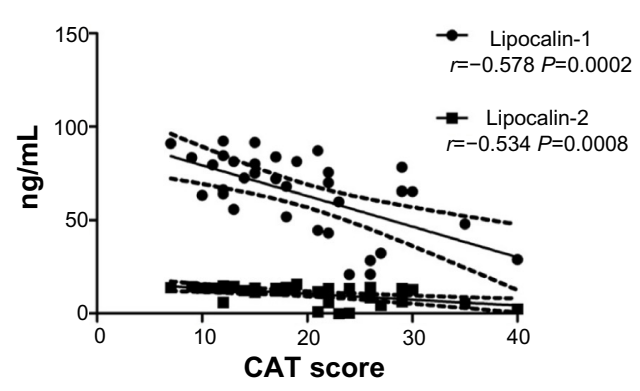

B

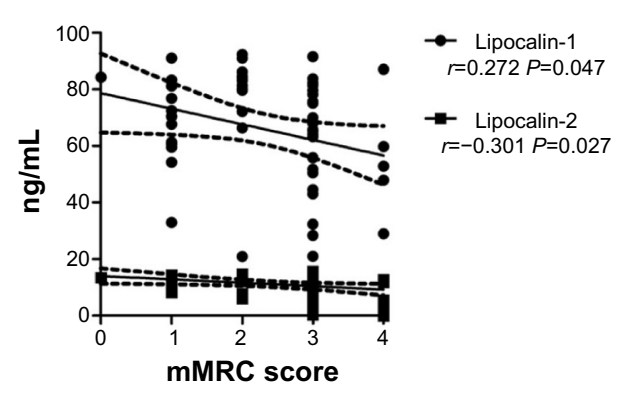

Figure 2 Serum levels of lipocalin-I and -2 in patients with COPD were both negatively correlated to CAT score (A) and mMRC score (B).

Abbreviations: CAT, COPD Assessment Test; COPD, chronic obstructive pulmonary disease; mMRC, modified Medical British Research Council dyspnea questionnaire.

cross-sectional evaluation, and therefore alterations in LCNs are correlative but not predictive. Besides, the medications for patients were not investigated, and the influence of inhaled corticosteroid on systemic levels of LCNs could not be measured.

\section{Conclusion}

We distinguished significant associations of circulating LCN1 and LCN2 with COPD and found a negative relationship with symptoms in patients with COPD. Large-scale and longitudinal studies are required to confirm our findings and validate the value of LCNs as biomarkers for COPD.

\section{Acknowledgment}

This study is supported by funding from Zhuoxue Talents Scheme of Fudan University, Shanghai Leading Talent Projects (No 036, 2010), Shanghai Committee of Science and Technology (Number 12411950100), and Shanghai Xuhui District Committee of Science and Technology (Number SHXH2013).

\section{Disclosure}

The authors report no conflicts of interest in this work.

\section{References}

1. Global Initiative for Chronic Obstructive Lung Disease. GOLD workshop report: global strategy for diagnosis, management and prevention of COPD [updated 2013]. Available from: http://www.goldcopd.org/Guidelines/ guidelines-global-strategy-for-diagnosis-management-2003-3.html. Accessed April 1, 2014.

2. Zhong N, Wang C, Yao W, et al. Prevalence of chronic obstructive pulmonary disease in China: a large, population-based survey. Am J Respir Crit Care Med. 2007;176:753-760

3. Fang X, Wang X, Bai C. COPD in China: the burden and importance of proper management. Chest. 2011;139:920-929.

4. Balkissoon R, Lommatzsch S, Carolan B, et al. Chronic obstructive pulmonary disease: a concise review. Med Clin North Am. 2011;95: $1125-1141$

5. Han MK, Agusti A, Calverley PM, et al. Chronic obstructive pulmonary disease phenotypes: the future of COPD. Am J Respir Crit Care Med. 2010;182:598-604.
6. Barnes PJ, Chowdhury B, Kharitonov SA, et al. Pulmonary biomarkers in chronic obstructive pulmonary disease. Am J Respir Crit Care Med. 2006;174:6-14.

7. Tzortzaki EG, Lambiri I, Vlachaki E, et al. Biomarkers in COPD. Curr Med Chem. 2007;14:1037-1048.

8. Woodruff PG. Novel outcomes and end points: biomarkers in chronic obstructive pulmonary disease clinical trials. Proc Am Thorac Soc. 2011;8:350-355.

9. Pinto-Plata VM, Mullerova H, Toso JF, et al. C-reactive protein in patients with COPD, control smokers and non-smokers. Thorax. 2006;61:23-28.

10. Eagan TM, Ueland T, Wagner PD, et al. Systemic inflammatory markers in COPD: results from the Bergen COPD Cohort Study. Eur Respir J. 2010;35:540-548.

11. Dickens JA, Miller BE, Edwards LD, et al. COPD association and repeatability of blood biomarkers in the ECLIPSE cohort. Respir Res. 2011;12:146.

12. Celli BR, Locantore N, Yates J, et al. Inflammatory biomarkers improve clinical prediction of mortality in chronic obstructive pulmonary disease. Am J Respir Crit Care Med. 2012;185:1065-1072.

13. Garcia-Rio F, Miravitlles M, Soriano JB, et al. Systemic inflammation in chronic obstructive pulmonary disease: a population-based study. Respir Res. 2010;11:63.

14. Lomas DA, Silverman EK, Edwards LD, et al. Serum surfactant protein D is steroid sensitive and associated with exacerbations of COPD. Eur Respir J. 2009;34:95-102.

15. Sin DD, Miller BE, Duvoix A, et al. Serum PARC/CCL-18 concentrations and health outcomes in chronic obstructive pulmonary disease. Am J Respir Crit Care Med. 2011;183:1187-1192.

16. Winkler C, Atochina-Vasserman EN, Holz O, et al. Comprehensive characterisation of pulmonary and serum surfactant protein D in COPD. Respir Res. 2011;12:29.

17. Dittrich AM, Meyer HA, Hamelmann E. The role of lipocalins in airway disease. Clin Exp Allergy. 2013;43:503-511.

18. Nicholas BL, Skipp P, Barton S, et al. Identification of lipocalin and apolipoprotein A1 as biomarkers of chronic obstructive pulmonary disease. Am J Respir Crit Care Med. 2010;181:1049-1060.

19. Lindahl M, Stahlbom B, Tagesson C. Newly identified proteins in human nasal and bronchoalveolar lavage fluids: potential biomedical and clinical applications. Electrophoresis. 1999;20:3670-3676.

20. Lechner M, Wojnar P, Redl B. Human tear lipocalin acts as an oxidativestress-induced scavenger of potentially harmful lipid peroxidation products in a cell culture system. Biochem J. 2001;356:129-135.

21. Seveus L, Amin K, Peterson CG, et al. Human neutrophil lipocalin (HNL) is a specific granule constituent of the neutrophil granulocyte. Studies in bronchial and lung parenchymal tissue and peripheral blood cells. Histochem Cell Biol. 1997;107:423-432.

22. Devarajan P. Review. Neutrophil gelatinase-associated lipocalin: a troponin-like biomarker for human acute kidney injury. Nephrology (Carlton). 2010;15:419-428. 
23. Flo TH, Smith KD, Sato S, et al. Lipocalin 2 mediates an innate immune response to bacterial infection by sequestrating iron. Nature. 2004;432:917-921.

24. Eagan TM, Damas JK, Ueland T, et al. Neutrophil gelatinase-associated lipocalin: a biomarker in COPD. Chest. 2010;138:888-895.

25. Cockayne DA, Cheng DT, Waschki B, et al. Systemic biomarkers of neutrophilic inflammation, tissue injury and repair in COPD patients with differing levels of disease severity. PLoS One. 2012;7:e38629.

26. Jessie K, Pang WW, Haji Z, et al. Proteomic analysis of whole human saliva detects enhanced expression of interleukin-1 receptor antagonist, thioredoxin and lipocalin-1 in cigarette smokers compared to non-smokers. Int J Mol Sci. 2010;11:4488-4505.
27. Redl B, Wojnar P, Ellemunter H, et al. Identification of a lipocalin in mucosal glands of the human tracheobronchial tree and its enhanced secretion in cystic fibrosis. Lab Invest. 1998;78:1121-1129.

28. Betsuyaku T, Nishimura M, Takeyabu K, et al. Neutrophil granule proteins in bronchoalveolar lavage fluid from subjects with subclinical emphysema. Am J Respir Crit Care Med. 1999;159:1985-1991.

29. Keatings VM, Barnes PJ. Granulocyte activation markers in induced sputum: comparison between chronic obstructive pulmonary disease, asthma, and normal subjects. Am J Respir Crit Care Med. 1997;155: $449-453$.

\section{Publish your work in this journal}

The International Journal of COPD is an international, peer-reviewed journal of therapeutics and pharmacology focusing on concise rapid reporting of clinical studies and reviews in COPD. Special focus is given to the pathophysiological processes underlying the disease, intervention programs, patient focused education, and self management protocols.

\section{Dovepress}

This journal is indexed on PubMed Central, MedLine and CAS. The manuscript management system is completely online and includes a very quick and fair peer-review system, which is all easy to use. Visit http://www.dovepress.com/testimonials.php to read real quotes from published authors.

Submit your manuscript here: http://www.dovepress.com/international-journal-of-copd-journal 